\title{
Iatrogenic Ureteral Transection During Suprapubic Cystolithotomy: A Rare Case Report
}

\author{
Mukesh Chandra Arya $^{1 *}$, Ankur Singhal ${ }^{2}$, Ajay Gandhi ${ }^{2}$, Yogendra Shyoran², Mahesh Sonwal ${ }^{2}$ and Rakesh \\ Singh ${ }^{2}$ \\ ${ }^{1}$ Professor and Head, Department of urology, Sardar Patel medical college, Bikaner, Rajasthan, India \\ ${ }^{2}$ Department of urology, Sardar Patel medical college, Bikaner, Rajasthan, India
}

*Corresponding author: Mukesh C Arya, Professor and Head, Department of

urology, Sardar Patel medical college, Bikaner, Rajasthan, India.

Received Date: October 13,2020

Published Date: December 22, 2020

\begin{abstract}
Primary vesical calculi are not uncommon in children in India. Percutaneous cystolithotomy (PCCL) or suprapubic cystolithotomy (SPCL) is the modality of treatment for large calculi owing to the small caliber of the urethra in children. Ureteral injury during SPCL is exceptional and unheard of. Herein, we report such a case with plausible reasons.
\end{abstract}

Keywords: Iatrogenic; Ureteral injury; Ureteric reimplantation; Suprapubic cystolithotomy

Abbreviations: PCCL: Percutaneous cystolithotomy; SPCL: Suprapubic cystolithotomy; DJ stent: Double J stent; SPC: Suprapubic Catheter

\section{Introduction}

Iatrogenic trauma during open surgery, laparoscopy, or endoscopic procedures is the most common cause of ureteral injury. Suture ligation, sharp incision or transection, avulsion, thermal injury and devascularization can cause ureteral damage. The majority of iatrogenic injuries involve lower ureter [1-3]. Injuries can be subtle and clinicians must have a high index of suspicion.

\section{Case Report}

A 6-year girl was referred to us after suprapubic cystolithotomy (SPCL) for a $2.5 \mathrm{~cm}$ primary vesical calculus (Figure 1). On day 3 with abdominal drain output more than $500 \mathrm{ml}$ per day with per urethral catheter draining $100 \mathrm{ml}$ per day. Her Hb was 9 gm\%, leucocyte count was 11300 per mm3 and serum creatinine was 1.1 mg\%. She was tested negative for Covid-19. On ultrasonography, her right kidney was small (54*26 mm), the left kidney (84*39 $\mathrm{mm}$ ) was normal in size with hypoechoic echotexture. The contrastenhanced CT scan (Figure 2) revealed prompt excretion of contrast from both kidneys. Also, mild hydroureteronephrosis was evident on the left side with contrast extravasation from the lower ureter.

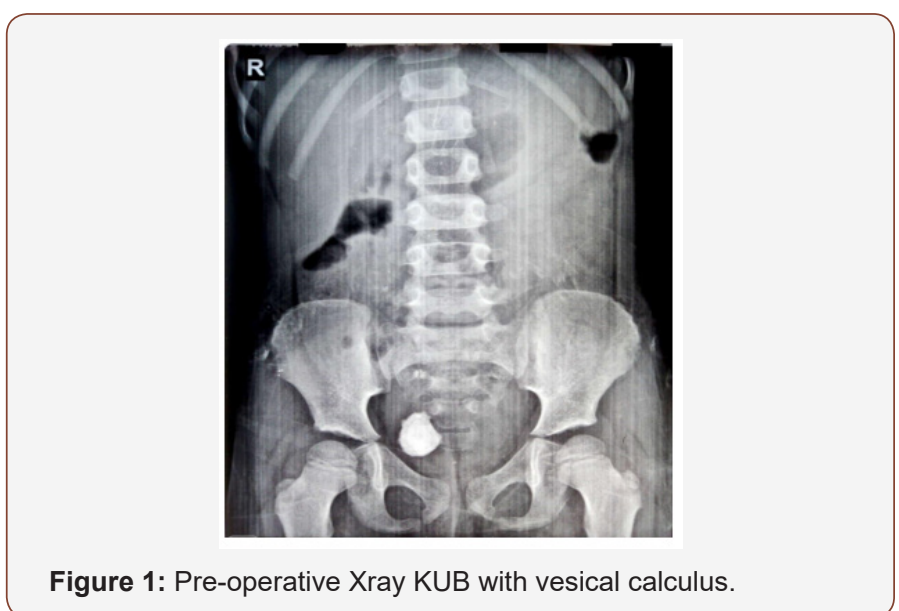

Figure 1: Pre-operative Xray KUB with vesical calculus. 


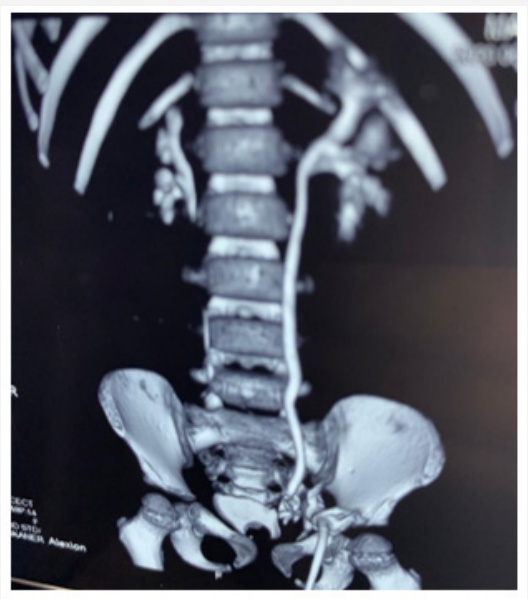

Figure 2: 3-D CT reconstruction image showing left hydroureteronephrosis with contrast extravasation from the lower ureter.

Under general anesthesia, left ureteroscopy revealed a complete transection of ureter $1.5 \mathrm{~cm}$ from the ureteric orifice. Since lower ureter was cut across, antegrade double J (DJ) stenting was not attempted. Exploration with lower midline abdominal incision was done. The left ureter was mobilized, and good length was obtained. To our utter surprise, anteriorly bladder wall was intact (Figure 3) and cystotomy was placed on the left posterolateral wall hardly $1 \mathrm{~cm}$ from ureteral hiatus (Figure 4). Even a retractor placed over ureter could transect it. Possibly, the assistant retracted the empty bladder to the right side and led to the judgmental error. We presume that with the distended bladder, it couldn't have happened. Through a separate anterior wall cystotomy, the intravesical ureter was excised. Distal ureteric margins were freshened and spatulated. Ureteric reimplantation was done into the same tunnel after putting a 4F DJ stent. Layered closure was done after putting $20 \mathrm{~F}$ malecot as a suprapubic catheter (SPC) and retropubic drain. She fared well postoperatively. Drain tube was removed on day 2 and was discharged on day 7 after the removal of stitches and SPC. She was advised to come 3 weeks later for DJ removal and was followed-up in the outpatient department with ultrasonography and micturating cystourethrogram at 3-months without no hydronephrosis or vesicoureteral reflux.

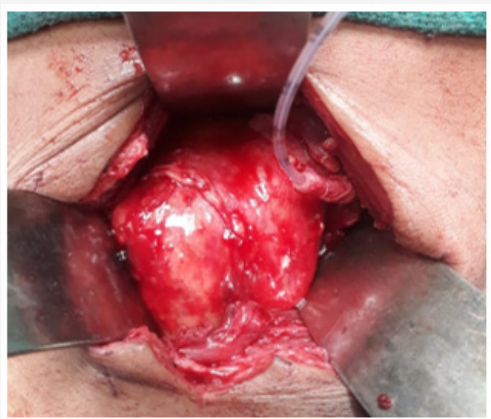

Figure 3: Intraoperative image with the intact anterior wall of the bladder with mobilized left ureter.

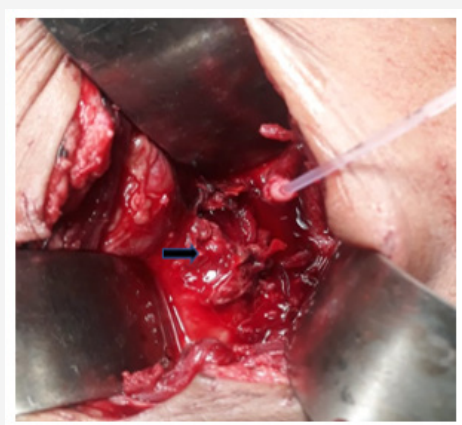

Figure 4: Intraoperative image showing the previous cystotomy on posterolateral wall. 


\section{Discussion}

The Majority of ureteral injuries occur during the hysterectomy, colorectal surgery, ovarian tumor removal, transabdominal urethropexy, abdominal vascular surgery, and radiation treatment $[4,5]$. If not treated, they can cause serious complications like urinoma, septicemia, renal failure and even loss of affected renal unit [6]. Management of such injuries depends upon location and severity of the ureteral injury. Ureteral injury during SPCL is unprecedented and it is unbelievable even in wildest dreams as to how it could happen. Left posterolateral cystotomy and manipulations close to the left ureteral hiatus led to its transection. Bladder filling with saline before cystotomy could prevent this complication. The opinion is divided over immediate reimplant and percutaneous nephrostomy and delayed repair [7]. Considering the only well-functioning left kidney and future pregnancy, anti-reflux ureteroneocystostomy was performed into the native tunnel.

\section{Conclusion}

The pelvic ureter is the most common site for iatrogenic ureteral injuries. Treatment depends upon the extent, cause, and timing of diagnosis. While ureteral reconstruction is a domain of open surgery, laparoscopic and robotic-assisted approaches are also being used. Ureteric transection during SPCL is an unusual and shocking complication. To our knowledge, it is not reported earlier.

\section{Conflict of Interest}

No conflict of interest.

\section{References}

1. Cadish LA, Ridgeway BM, Shepherd JP (2019) Cystoscopy at the time of benign hysterectomy: a decision analysis. Am J Obstet Gynecol 220(4): 369.e1-369.e7.

2. Li X, Li H, Li C, Luo X, Song Y, et al. (2019) Migration of an intrauterine device causing severe hydronephrosis progressing to renal failure: A case report. Medicine (Baltimore) 98(3): e13872.

3. Douissard J, Meyer J, Ris F, Liot E, Morel P, et al. (2019) Iatrogenic ureteral injuries and their prevention in colorectal surgery: results from a nationwide survey. Colorectal Dis 21(5): 595-602.

4. Tyritzis SI, Wiklund NP (2015) Ureteral strictures revisited trying to see the light at the end of the tunnel: a comprehensive review. J Endourol 29: $124-136$.

5. Abboudi H, Ahmed K, Royle J, Khan MS, Dasgupta P, et al. (2013) Ureteric injury: a challenging condition to diagnose and manage. Nat Rev Urol 10: $108-115$.

6. Lucarelli G, Ditonno P, Bettocchi C, Grandaliano G, Gesualdo L, et al. (2013) Delayed relief of ureteral obstruction is implicated in the longterm development of renal damage and arterial hypertension in patients with unilateral ureteral injury. J Urol 189: 960-965.

7. Engelsgjerd JS, LaGrange CA (2020) Ureteral Injury. [Updated 2020 May 30]. In: StatPearls [Internet]. Treasure Island (FL): StatPearls Publishing.

\section{Acknowledgment}

None. 